Running head: RUINATION AND INTERNAL SWITCHING OF ATTENTION

\title{
Rumination is characterized by valence-specific impairments in switching of attention
}

Ernst H.W. Koster, Evi De Lissnyder, \& Rudi De Raedt

Department of Experimental-Clinical and Health Psychology

Ghent University, Belgium

Reference: Koster, E.H.W., De Lissnyder, E., \& De Raedt, R. (in press). Rumination is characterized by valence-specific impairments in internal shifting of attention. Acta Psychologica.

Disclaimer: This is an uncorrected proof and might still change from the final journal version.

* Address for correspondence: Ernst Koster, Ghent University, Department of Psychology, Henri Dunantlaan, 2, B-9000 Ghent, Belgium. Tel: +0032 0926464 46, fax: +0032 09264 64 89, e-mail address: Ernst.Koster@UGent.be 


\begin{abstract}
The ability to flexibly switch attention between emotional and non-emotional information in working memory is considered important in stress-resilience and is impaired in mood disorders. A recent theory claims that this component of attention is specifically related to ruminative thought. To further investigate this claim we report two new experiments using the internal shift task (IST). In experiment $1(N=27)$ we examined the stability of switching ability measured using the IST through examination of internal consistency (stability within the task) and test-retest-reliability (stability over time) over two weeks. Results indicate relatively high stability of switching ability measured with the IST. In experiment 2 the IST was administered to a pre-selected undergraduate sample of high $(N=20)$ and low ruminators $(N=20)$. The main findings were that rumination was related to attentional switching impairments, specifically in the context of emotional information. The switching impairments were most pronounced when negative information was held in working memory. The attentional switching impairments were most strongly related to the depressive brooding component of rumination. The results of this study lend further support to the proposed link between rumination and switching abilities.
\end{abstract}

Keywords: attentional control, switching, rumination, depression, working memory 


\section{Introduction}

Persistent negative thought is considered a hallmark feature of affective disorders (Nolen-Hoeksema, Wisco, \& Luybomirsky, 2008; Watkins, 2008). A well-investigated form of persistent negative thought - which is related to negative affect and depression - is rumination. Rumination is defined as "behaviours and thoughts that focus one's attention on one's depressive symptoms and on the implications of those symptoms" (Nolen-Hoeksema, 1991, p. 569). Two distinct subtypes of rumination are distinguished (Treynor, Gonzalez, \& Nolen-Hoeksema, 2003). The first, reflective pondering, is considered a more adaptive form of rumination and reflects the degree to which individuals engage in cognitive problem solving to improve their mood. The second, depressive brooding, is considered a more maladaptive form of rumination and reflects the degree to which individuals passively focus on symptoms of distress and the meaning of those symptoms (Nolen-Hoeksema et al., 2008). The brooding component is proposed to be most closely related to depression risk (Treynor et al., 2003). In general, it has been shown that rumination, especially brooding, has detrimental effects on affect and cognition and acts as a crucial vulnerability factor for depression (NolenHoeksema et al., 2008)

An important question is why individuals persist in this thinking style despite its negative consequences. Recently, several authors have proposed that impaired attentional control contributes to ruminative thinking (Hertel, 1997; Koster, De Lissnyder, Derakshan, \& De Raedt, 2011; Joormann \& D’Avanzato, 2010; Linville, 1995). The latter proposal has generated a wealth of research examining different aspects of attentional control in relation to persistent negative thought, negative mood, and psychopathology (e.g., Joormann, Yoon \& Zetschke, 2007; Whitmer \& Banich, 2007). Remarkably, depression-related research is suggesting that attentional impairments and biases are more proximinally related to rumination than to the broad construct of depression (Koster et al., 2011). 
Research on depression and rumination has widely examined attentional processes in the processing of neutral and affective information. In the context of depression, attentional impairments in the processing of neutral material are mainly observed in individuals with severe levels of depression (e.g., Rokke, Arnell, Koch, \& Andrews, 2002). Interestingly, when processing mood-congruent information attentional impairments, mainly at the level of attentional disengagement, can also be observed in subclinically depressed samples (De Raedt \& Koster, 2010). Rumination has also been linked to attentional impairments. Davis and Nolen-Hoeksema (2000) showed using the Wisconsin Card Sorting Test that ruminators are characterized by cognitive inflexibility. At the level of attention, in an antisaccade task containing neutral material, where participants are instructed to generate a saccade to the mirror position of an abrupt peripheral cue, ruminators showed impaired inhibition as indicated by slower antisaccade latencies (De Lissnyder, Derakshan, De Raedt, \& Koster, 2011). These inhibition impairments were specifically related to depressive brooding. In the context of emotional stimuli, rumination has been linked to biased attention towards negative words (Donaldson, Lam, \& Mathews, 2007). This study was conducted in a sample of depressed patients and showed that trait rumination was associated with an attentional bias for negative words, even when depressive symptoms were statistically controlled for.

Recently, Chun Golomb, and Turke-Brown (2011) proposed a distinction between external and internal attention, where external attention refers to processing of external information and internal attention refers to processing information or mental operation on stimuli that are held in memory. It is noteworthy that both in depression and rumination, studies have mainly examined attentional processing of external information (pictures, faces, or words), whereas depression and rumination may be characterized by impaired internal attention over negative thoughts and memories. In this context, Garavan (1998) and Gehring et al. (Gehring, Bryck, Jonides, Albin, \& Badre, 2003) developed a paradigm to switching 
between objects held in working memory. Recently, Chambers, Lo, and Allen (2008) developed an affective version of this paradigm using words as stimuli. We further modified this task to include emotional facial expressions and refer to this task as the Internal Shift Task (IST; De Lissnyder, Koster, \& De Raedt, 2012). In the IST individuals are required to perform a mental count based on the emotional expression of a face (i.e., count the number of negative and neutral faces) in one block (referred to as emotion condition) or on nonemotional features of a face (i.e., count the number of males and females) in another block (referred to as gender condition). Interestingly, the IST allows to examine differential switching capacity ${ }^{1}$ in relation to specific task settings (emotion versus gender condition), valence-specific impairments (within the emotion condition: switching from negative to neutral or vice versa) and general switching capacity (across both emotion and gender conditions).

In previous research in dysphoric participants using the IST we observed that attentional control impairments were not related to depression scores but were related to rumination scores (De Lissnyder et al., 2012). Similar effects were obtained by, among others, Whitmer and Banich (2007) who investigated inhibition as well as set shifting related to different types of rumination. In a task switching paradigm that distinguished inhibition and set shifting impairments in the context of non-emotional information, they found that brooding, but not depression, scores were specifically associated with impaired inhibition. Since then, similar findings have been obtained in a variety of task suggesting that rumination is more strongly correlated with attentional impairments than depression scores (for a review, see Koster et al., 2011). Moreover, a recent study measuring event-related potentials during the IST (containing words) indicates that ruminators need to exert more effort in switching when they are in a negative mood state (Yee Lo, Lau, Cheung, \& Allen, 2012)

\section{Present Study}


Internal attention appears to be strongly correlated with rumination, where the research using the IST to investigate whether the ability to switch between (affective) mental representations is associated with the ability to control (negative) thoughts in rumination seems particularly relevant. However, there are important drawbacks of previous research. First, little is known about the stability of the switching ability scores obtained in the IST. Generally, in this research it is assumed that switching abilities obtained at a single time point represent a stable trait related to attentional control in general. However, at present there is little data to support this claim. As little information is available on affective modifications of attention paradigms, with the processing of emotional information being potentially more susceptible to state influences, research on this issue is imperative. Therefore, in a first experiment, the IST was administered to an unselected undergraduate sample $(N=27)$ to examine the internal consistency (stability within the task) and test-retest reliability (stability over time) over two weeks of the IST.

Second, in our previous research using the IST (De Lissnyder et al., 2012) we selected dysphoric individuals and included analyses based on depression as well as rumination scores based on a median split procedure for rumination. In that research it was found that rumination was associated with impaired switching in the emotional task condition of the IST (where individuals had to categorize angry and neutral facial expression). However, we failed to find the expected valence-specific differences in switching away from angry versus neutral faces. This is in contrast to much attentional research where impaired disengagement was found for negative information (for a review, see De Raedt \& Koster, 2010). Provided that individuals were pre-selected on depression scores and individuals were allocated to a high and low rumination through median-split, it may be better to examine attentional control in pre-selected high and low rumination groups. Thus, in experiment 2 , we sought to replicate 
and extend our investigation into valence-specific switching impairments in an undergraduate sample of high ruminators $(N=20)$ and low ruminators $(N=20)$.

In sum, the following hypotheses were tested in each of the respective experiments:

(1) If switching ability between mental representations indeed is a stable individual difference variable, scores on the IST should show high internal consistency and high testretest reliability in Experiment 1;

(2) If rumination is related to valence-specific switching impairment, high ruminators should show longer RT to switch from angry to neutral faces in the emotional task condition compared with low ruminators.

\section{EXPERIMENT 1: STABILITY OF IST PERFORMANCE}

\section{Method}

\section{Participants}

The initial sample included 30 undergraduates, three participants were excluded from analysis due to missing retest data (failure to show up). The final sample included 27 participants ( 9 males, 18 females) ranging in age from 18 to 26 years $(M=20.63, S D=1.78)$. Participants were paid ( 8 euro) for their contribution. The study was approved by the ethical committee at Ghent University.

\section{Materials}

\section{Self-report questionnaires}

\section{Rumination}

The Ruminative Response Scale (RRS-NL) was used to measure rumination (NolenHoeksema \& Morrow, 1991; Raes et al., 2009). The RRS-NL is a 26-item self-report measure and consists of items that describe responses to a depressed mood that are focused on the self, 
symptoms, or consequences of depressed mood. Participants are requested to indicate how often they engage in these responses using a four-point Likert scale ranging from 1 (almost never) to 4 (almost always). Total rumination scores range from 26 to 104 . A factor analysis of the RRS has identified two separate subscales that are differentially related to depressive symptoms, reflective pondering and depressive brooding. The RRS is a reliable and valid measure of rumination with good psychometric properties (Treynor et al., 2003).

\section{Depressive symptoms}

The Beck Depression Inventory - Second Edition (BDI-II) was used to measure depressive symptoms (Beck, Steer, \& Brown, 1996; Van Der Does, 2002). The BDI-II is a 21-item self-report measure with robust psychometric properties (Beck, Steer, \& Garbin, 1988) which assesses the severity of a range of affective, somatic and cognitive symptoms of depression. Individuals rate each symptom on a scale ranging from 0 to 3 and scores on the BDI-II could range from 0 to 63 .

\section{Internal Shift Task}

\section{Design}

The task was programmed using E-prime 2.0 software package and ran on a Windows XP computer with a $75 \mathrm{~Hz}, 19$-inch colour monitor.

The faces presented in the IST were taken from the Karolinska Directed Emotional Faces (KDEF) (Lundqvist, Flykt, \& Öhman, 1998). All faces were adjusted to exclude interference of background stimuli (hair) and were adjusted to the same size (326 x 326 pixels $)$. Based on intensity $(1=$ not at all $-9=$ completely $)$ and arousal $(1=$ calm $-9=$ aroused) ratings, a total of 24 neutral (Intensity: $M=5.15, S D=0.37$; Arousal: $M=2.48, S D$ $=0.23$ ) and 24 angry (Intensity: $M=6.36, S D=0.71$; Arousal: $M=3.87, S D=0.58$ ) faces were selected from a validation study of the KDEF picture set (Goeleven, De Raedt, Leyman, \& Verschuere, 2008). Angry facial expressions were chosen as stimulus material because they 
convey direct information concerning interpersonal evaluation, which is of high relevance to depression and rumination (Gotlib \& Hammen, 2002) which are characterized by fear of social rejection (Barnett \& Gotlib, 1988).

In the IST, faces were presented at the centre of the computer screen one at a time. All participants were asked to complete two task conditions, a non-emotional condition (hereafter referred to as gender condition) and an emotional one (hereafter referred to as emotion condition). The two conditions (emotion and gender) were completed sequentially and the order in which the conditions were completed was counterbalanced across subjects. In the gender condition, participants had to focus on the 'gender' dimension of the face (the faces had to be categorized as male or female), whereas in the emotion condition, they had to focus on the 'emotion' dimension of the face (the faces had to be categorized as neutral or angry). There were 12 blocks of trials for both conditions with random 10 to 14 presented faces within each block. The participant's task was to keep a silent mental count of the number of faces in each category, presented within a block of trials (e.g., participants had to update counters for male and female faces in the gender condition; participants had to update counters for neutral and angry faces in the emotion condition). When a face was presented, participants were asked to press the spacebar as fast as possible (reaction time measure) to indicate that they had updated both internal counters. The next face appeared on the screen after a 200ms inter-trial interval. Participants had to report the number of faces for both categories (accuracy measure), using the number path of the keyboard, at the end of each block in a fixed order to encourage a consistent counting strategy (e.g., in the emotion condition they had to report their counts first for the neutral and then for the angry faces, in the gender condition the order was male-female). Due to the sequence of the faces, there were switch and no switch trials in each block of items. Switch costs were calculated as the difference in reaction time between switch and no-switch trials within the blocks and served 
as the main dependent variable in the analyses. A trial is regarded as a switch trial if a target trial $(n)$ has to be updated on a different category as its preceding trial $(n-1)$ (i.e., in the emotion condition: angry-neutral and neutral-angry). A trial is regarded as a no-switch if a target trial $(n)$ has to be updated on the same category as its preceding trial (n-1) (i.e., in the emotion condition: angry-angry and neutral-neutral). In addition, due to the task design, valence-specific emotional switching effects could be investigated (i.e., comparing the switches angry-neutral versus neutral-angry in the emotion condition). The practice trials consisted of 3 blocks of items and the experimental trials of 12 blocks of items in each condition. An example of a block of items and stimulus display is presented in Figure 1.

(Figure 1 about here)

\section{Data-analytic approach of the IST}

For the analyses of reaction times, median scores were used to reduce any influence of outliers in the within-subject data. All blocks of items, correct and incorrect, were included in the data-analyses. A block of items was considered correct only if both reported numbers of the faces in each category were accurate. Responding in most blocks was highly accurate (test 1: $93 \%$; test $292 \%$ ). Inclusion of correct and incorrect blocks has the advantage of providing similar numver of observations within each cell of the design.

To obtain a measure of switching capacity between internal representations held in working memory, switch costs were calculated. The measurement of switch costs is held to be of interest because, as is widely believed, they reflect the control processes that are engaged when subjects switch between multiple tasks, operations or mental sets. In the switching literature, the reaction time (RT) switch cost is typically referred to as the difference in RT between switch and no-switch (or repeat) trials (Monsell, 1996).

First, the IST allows us to assess general switching capacity in working memory across both emotion and gender conditions (hereafter reaction time indices are referred as 
general switch, general no-switch, general switch cost). Second, the IST allows us to assess switching capacity within conditions, in particular for emotional (emotion condition) and nonemotional (gender condition) information (hereafter reaction times indices are referred as switch emotion, no-switch emotion, switch cost emotion, switch gender, no-switch gender, switch cost gender). Finally, the IST allows us to examine the switches and no-switches within the emotion condition (hereafter reaction time indices are referred as angry-neutral or neutral-angry versus angry-angry or neutral-neutral for the emotion condition)

\section{Procedure}

Participants performed the IST twice in individual experimental sessions, with a testretest-interval of two weeks. In the first session, the IST was administered after the participants completed an informed consent form. Participants were asked to perform the IST as quickly and accurately as possible. They practiced the IST and then completed the experimental session consisting of 12 blocks of items in both conditions, with a short break in between the emotion and gender condition. At the end of the experiment all participants were fully debriefed.

\section{Results}

Estimates of both internal consistency and the test-retest-reliability over two weeks of the reaction time based response indices are shown in Table 1. The split-half method was used for estimating the internal consistency (stability within the task) of the reaction time indices. The split-half reliability was computed by splitting both conditions into two test halves (even and odd trials) and the different reaction time indices of each test half were correlated (Spearman-Brown correlation coefficient). The retest-reliability (stability over time) was determined by computing correlations of the same reaction time indices measured over two weeks. 
The split-half correlations ranged from, $r(27)=.15, p<.01$, to, $r(27)=.90, p<.01$, with most correlations $>.4$. Moreover, test-retest correlations were all $>.5$. Finally, the results also revealed a significant correlation of the accuracy on the IST over the two test moments, $r(27)=.53, p<.01\left(M^{\text {test }}=93 \%, S D^{\text {test }}=6 \% ; M^{\text {restest }}=92 \%, S D^{\text {restest }}=8 \%\right)$.

(Table 1 about here)

\section{Discussion}

In this first experiment, the IST was administered to an unselected sample to examine the stability of IST performance. Estimates of both internal consistency and the test-retestreliability over two weeks indicate that switching ability as measured by the IST is a relatively stable phenomenon. That is, the results revealed that the split-half correlations were sufficient. Moreover the results revealed that all test-retest correlations were significant and quite high. Most importantly, the test-retest correlations of the most crucial dependent variables for our research purposes (switch cost reaction time indices), were all very high (between .85 and .91). Estimates of the split-half correlations were generally lower as compared to test-retest reliability coefficients. The reason for this difference might still be that reliability estimates based on a split-half procedure has less power because it is calculated on half of the items, causing error variance to potentially exert a larger influence on performance indices. In addition, the split-half correlations of the specific switches and no-switches within conditions (e.g., angry-angry) were lower than for the higher-order estimates (switch and no switch). This was to be expected, as these are very specific analyses of reaction time responses within trials. Importantly, the test-retest reliability of these specific switches and no-switches still was sufficient. Finally, we have no clear explanation for the low split-half correlation of the neutral-neutral no-switch trials. It may be that some of these neutral facial expressions were perceived as somewhat ambiguous. Still, this latter finding did not hamper the test-retest reliability of the overall task. 
Provided that previous research has indicated that attentional indices obtained in reaction time tasks that contain emotional information have very poor test-retest reliability (e.g., emotional stroop: < .3; Kindt, Bierman, \& Brosschot, 1996; dot probe task: < .1; Schmukle, 2005), the obtained reliability scores (all test-retest indexes: > .5) can be considered high.

\section{EXPERIMENT 2: RUMINATION AND SWITCHING ABILITY}

\section{Method}

\section{Participants}

In this experiment, 40 undergraduates of Ghent University, 39 females and 1 male ranging from 18 to 26 years in age $(M=19, S D=2)$, participated in return for credits or financial compensation $(8 €)$. They were recruited by means of an on-line participant panel system. Participants completed the RRS-NL as a screening measure. Based on the total RRSNL scores, participants who scored in the highest quartile were classified as high ruminators $(N=20)$, participants who scored in the lowest quartile were classified as low ruminators $(N=$ 20). Upon invitation for the experiment, they completed the RRS-NL again. By design, the high rumination group had significantly higher RRS-NL scores $(M=65, S D=9)$ compared to the low rumination group $(M=41, S D=7), t(38)=9.35, p<.001$. Age $(t<1)$ in both groups was not significantly different. Note that the number of dysphoric participants (BDI-II > 13, cf. Beck et al., 1996) was not distributed evenly over both rumination groups, $\chi^{2}(1, N=40)=$ $10, p<.01$, with 8 dysphoric participants in the high rumination group, 0 dysphoric participants in the low rumination group, 20 non-dysphorics in the low rumination group and 12 non-dysphorics in the high rumination group. The mean BDI-II score was significantly different in the high $(M=10.9)$ compared with the low ruminator group $(M=2.7), \mathrm{t}(38)=$ $4.38, \mathrm{p}<.01)$

\section{Materials and Procedure}


Materials and procedure were kept similar to experiment 1, with the exception that in experiment 2 the IST was administered only once. Moreover, participants additionally completed the trait version of the Spielberger State-Trait Anxiety Inventory (STAI-T; Spielberger, 1983; Dutch version: Van der Ploeg, Defares, \& Spielberger, 2000). This 20-item questionnaire measures trait anxiety or the tendency to respond with anxiety to perceived threats. The reliability and validity of the STAI-T is well-documented in healthy as well as clinical samples (Spielberger, 1983; Van der Ploeg et al., 2000). As depression and anxiety levels typically correlate highly this allows to examine whether effects are related to rumination, depression, or anxiety levels. The study was approved by the ethical committee at Ghent University.

\section{Results}

\section{Emotion versus gender condition}

Analyses revealed an average accuracy rate of $88 \%$. There was a significant difference in error rate between the two conditions, $F(1,38)=6.84, p<.05$, with higher error rates in the gender condition $(M=15 \%, S D=13 \%)$ compared to the emotion condition $(M=9 \%, S D=$ $9 \%)$. There was no significant difference in error rate between both groups, $F(1,38)=2.50, p$ $>$.1. The condition $\mathrm{x}$ group interaction on the error data was not significant, $F<1$.

(Table 2 about here)

Overall means and standard deviations for the different trial types can be found in Table 2. A mixed ANOVA on reaction times with Condition (emotion, gender) and Switch Type (switch, no-switch) as within-subject factors and Group (low ruminators, high ruminators) as between-subject factor, revealed a main effect of Switch Type, $F(1,38)=$ $183.53, p<.001$, with reaction times on switch trials $(M=1501 \mathrm{~ms}, S D=272 \mathrm{~ms})$ being slower than reaction times on no-switch trials $(M=1161 \mathrm{~ms}, S D=226 \mathrm{~ms})$. Analyses also revealed a Condition $\mathrm{x}$ Switch Type interaction, $F(1,38)=4.57, p<.05$. Importantly, analyses 
revealed a significant Condition $\mathrm{x}$ Switch Type $\mathrm{x}$ Group interaction, $F(1,38)=5.93, p<.05$. Within group comparisons showed that for the high ruminators the switch cost in the emotion condition was significantly higher $(M=425 \mathrm{~ms}, S D=218 \mathrm{~ms})$ than in the gender condition $(M$ $=351 \mathrm{~ms}, S D=190 \mathrm{~ms}), t(19)=3.14, p<.01$. This difference in switch cost between both conditions was not significant in the low ruminators, $t<1$. Between group comparisons showed that the switch cost in the emotion condition was larger in the high rumination group $(M=425 \mathrm{~ms}, S D=218 \mathrm{~ms})$ compared to the low ruminators $(M=302 \mathrm{~ms}, S D=116 \mathrm{~ms}), t(38)$ $=2.22, p<.05$. There was no difference between groups for the gender condition, $t(38)<1$. These results on switching in function of rumination group are depicted in Figure 2.

(Figure 2 about here)

Controlling for Depression and Anxiety Level. To examine the relative contribution of rumination and depression scores to switching abilities, we performed a hierarchical regression analysis with the switch cost in the emotion condition as dependent variable and scores for depressive symptoms (BDI-II) as predictor in a first step and depressive rumination or brooding and reflective pondering (RRS) as predictors in the second step ${ }^{2}$. The zero-order correlations of the variables that were entered in the regression analyses (across all participants) are presented in Table 3. Depressive symptoms did not predict switch costs but the second step added significantly to the prediction, $F(2,27)=3.53, p<.05, R^{2}$ change $=.19$. The only variable that significantly predicted switching in the emotion condition was depressive brooding, $\beta=-.51, t=2.57, p<.05$. Higher scores on depressive brooding were associated with impaired switching in the context of emotional information, reflected in larger switch costs in the emotion condition.

(Table 3 about here)

Given the high correlation between depression and anxiety scores in the full sample $(r$ $=.76, \mathrm{p}<001$, we repeated the above analysis but now with anxiety as first step instead of 
depression scores. This analysis showed that anxiety did not predict switch costs but the second step added significantly to the prediction, $F(2,27)=4.07, p<.05, R^{2}$ change $=.19$, similar to the initial regression analysis.

\section{Valence-specificity within the emotion condition}

To further investigate valence-specific attentional impairments exclusively on trials in the emotion condition, performance on switch trials (angry-neutral, neutral-angry) and noswitch trials (angry-angry, neutral-neutral) was investigated. A mixed ANOVA on RT of trial $n$ with Valence Face $n-1$ (neutral, angry) and Valence Face $n$ (neutral, angry) as within subject factors and Group (low ruminators, high ruminators) as between-subject factor revealed a significant main effect of Valence Face $n-1, F(1,38)=6.04, p<.05$. Responses on angry $n-1$ faces $(M=1358 \mathrm{~ms}, S D=242 \mathrm{~ms})$ were slower than responses on neutral $n-1$ faces $(M=$ $1327 \mathrm{~ms}, S D=242 \mathrm{~ms})$. Analyses revealed a significant main effect of Valence Face $n, F(1,38)$ $=170.12, p<.001$. Responses on neutral $n$ faces $(M=1359 \mathrm{~ms}, S D=253 \mathrm{~ms})$ were slower than responses on angry $n$ faces $(M=1325 \mathrm{~ms}, S D=230 \mathrm{~ms})$. Analyses revealed a significant Valence Face $n$ and Group interaction effect, $F(1,38)=4.22, p<.05$, and Valence Face $n-1 \mathrm{x}$ Face $n$ interaction, $F(1,38)=7.66, p<.01$. Importantly, these two-way interactions can be subsumed under a significant three-way interaction effect, $F(1,38)=4.33, p<.05$. Means for this interaction are displayed in Figure 3. To further corroborate the three-way interaction we performed separate ANOVAs for no-switch and switch trials.

(Figure 3 about here)

For the no-switch trials (angry-angry versus neutral-neutral), analyses revealed no significant Valence Face $n-1 \times$ Group interaction, $F<1$. For the switch trials (angry-neutral versus neutral-angry), analyses revealed a significant Valence Face $n-1$ x Group interaction, $F(1,38)=6.20, p<.05$. Within group comparisons revealed that for the high ruminators responses on switch angry-neutral trials $(M=1647 \mathrm{~ms}, S D=292 \mathrm{~ms})$ were slower than 
responses on switch neutral-angry trials $(M=1533 \mathrm{~ms}, S D=244 \mathrm{~ms}), t(19)=3.74, p<.001$. This difference was not significant in the low ruminators, $t<1$. Between group comparisons showed that responses on angry-neutral trials were slower in the high rumination group $(M=$ $1647 \mathrm{~ms}, S D=292 \mathrm{~ms})$ compared to the low ruminators $(M=1463 \mathrm{~ms}, S D=259 \mathrm{~ms}), t(38)=$ $2.11, p<.05$. There was no difference between groups for the neutral-angry trials, $t(38)<1$. The results are depicted in Figure 3.

Controlling for Depression and Anxiety Level. We performed a hierarchical regression analysis with the valence-specific switching impairment as dependent variable and scores for depressive symptoms (BDI-II) as predictor in the first step and depressive rumination and reflective pondering (RRS) as predictors in the second step. The zero-order correlations of the variables that were entered in the regression analyses are presented in Table 4. Depressive symptoms did not predict switch costs but the second step added significantly to the prediction, $F(2,27)=4.33, p<.05, R^{2}$ change $=.23$. The only variable that significantly predicting switching in the emotion condition was depressive brooding, $\beta=-.58, t=2.92, p$ $<.05$. Higher scores on depressive brooding were associated with a valence-specific switching impairment, reflected in slower responses when switching from an angry to a neutral face.

Again, we repeated the above analysis but now with anxiety as first step instead of depression scores. This analysis showed that anxiety did not predict switch costs but the second step added significantly to the prediction, $F(2,27)=4.85, p<.05, R^{2}$ change $=.25$, similar to the initial regression analysis.

(Table 4 about here)

\section{Discussion}

In this second experiment, the IST was administered to a pre-selected undergraduate sample of high ruminators $(N=20)$ and low ruminators $(N=20)$ to examine switching 
impairment in rumination. The results indicate that rumination was related to switching impairments in the context of emotional information. The switching impairments were most pronounced when switching from negative to neutral information. The rumination component that was most predictive of the switching impairments was depressive brooding. However, it is noteworthy that the findings obtained with rumination were not fully independent from depression level.

\section{General Discussion}

The research presented in this article extends research on the link between attentional control, rumination, and depression. Using the IST we examined whether switching ability, as measured with the IST, is a stable cognitive characteristic and whether rumination is associated with valence-specific switching impairments. Results suggest that (1) switching ability as measured with the IST is a relatively stable phenomenon; (2) rumination is associated with impaired switching especially from negative information; (3) switching impairments are mainly related to brooding scores. These results are discussed in turn below.

In the first experiment, the IST was administered to an unselected sample to examine the stability of IST performance. This is relevant as in most research it is assumed that scores on tasks measuring aspects of attentional control represent stable ability scores. However, in many tasks, especially affective modifications, this notion has not been tested and it is possible that such task are sensitive to state influences and/or that RTs generated by such tasks have a high error variance (due to influences of among others motor preparation and responding). In attention tasks where reliability has been tested, results have often been disappointing (test-retest reliability $<.3$ ). Our results indicate that within one session the various RT indices of the IST generally have sufficient to high internal consistency. Moreover, the two week test-retest reliability of the IST is adequate to high for all the crucial indices. 
Thus, experiment 1 indicated that scores on switching ability or relatively stable. Clearly it should be noted that many of the test circumstances were kept similar and the time between two test sessions was not very long (two weeks). Therefore, these data should not be taken as evidence to conceive of measuring attentional control as a trait variable comparable to questionnaire research. Obviously, attentional control is a dynamic process with performances being strongly determined by specific circumstances. Still, it is reassuring to find that ability scores on a RT paradigm such as the IST, are relatively stable as this is not generally the case for paradigms used in experimental psychopathology research (e.g., the dot probe task, Schmukle, 2005). It was an important first step to establish the stability of switching ability in a healthy sample as this may be more difficult in a sample with high levels of psychopathology where there is often more mood fluctuation. The high test-retest reliability suggests that results should be relatively easy to replicate and that the IST is suitable for research where multiple administration of the task is required.

In the second experiment, the IST was administered to a pre-selected undergraduate sample of high and low ruminators to examine valence-specific switching impairments. In recent years, research has found evidence showing that rumination is negatively correlated with attentional control (Davis \& Nolen-Hoeksema, 2000; De Lissnyder, Koster, Derakshan, \& De Raedt, 2010; De Lissnyder et al., 2012; Joormann, 2006; Whitmer \& Banich, 2007). The second experiment in this study improves upon previous studies for several reasons. First, attentional control has been mainly examined in relation to depressive symptoms in general, with only limited research on rumination (but see Joormann, Levens, \& Gotlib, 2011). Second, to date, research on attentional control in rumination has mainly used non-emotional stimuli (Davis \& Nolen-Hoeksema, 2000; Whitmer \& Banich, 2007). This is an important limitation as previous research indicates that emotion processing is more severely impaired than information processing in general (Joormann et al., 2007). Finally, studies have 
examined the relation between rumination and attentional control when confronted with emotional information (De Lissnyder et al., 2010), but attentional control was mainly examined in relation to externally presented stimuli. The second experiment in this study focused on examining if rumination is related to impaired attentional control over internal representations of emotional and non-emotional information held in working memory. The results indicate that rumination was related to internal switching impairments, specifically in the context of emotional information. The switching impairments were most pronounced when switching from negative to neutral information. These results are largely in line with previous research using the IST in a pre-selected sample of dysphoric and non-dysphoric participants (De Lissnyder et al., 2012). However, the results of the current experiment where we selected on rumination scores showed more pronounced valence-specific effects compared with our previous study. We performed hierarchical regression analyses to examine when effects of rumination are still observed when controlling for depression (and subsequently anxiety) levels in a first step. We consistently found that brooding scores, but not depression, anxiety, and reflection scores, predicted the switching impairments. This is in line with previous research examining attentional control in relation to depression as well as rumination (Bernblum \& Mor, 2010; De Lissnyder et al., 2010; De Lissnyder et al., 2012), where distinct relations were found between the two types of rumination and information processing.

Some limitations of the current study need to be mentioned. First, although experiment 1 suggests that the IST has good test-retest reliability, this experiment was conducted in a small sample and cannot serve as a psychometric evaluation of the IST as this would require a larger sample size. Moreover, we examined reliability of the IST in a relatively homogeneous undergraduate sample. Thus, at present we are not able to conclude that the IST will also be a reliable measure in clinical samples. Moreover, in the IST we only presented angry faces as emotional stimuli. Although we found switching impairments for angry versus neutral faces 
we cannot conclude that the impairments are restricted to angry or even negative material as no other emotional facial expressions (e.g., sad, happy faces) were included. Another issue is that our conclusions with regard to IST performance and rumination are restricted to females as there only was one male participant in experiment 2 . Provided that there are important gender differences in ruminative tendencies (e.g., Nolen-Hoeksema et al., 2008), further investigation of the underlying cognitive mechanisms involved in rumination in relation to gender would be interesting. Finally, although we have tried to examine the relative contribution of depression, anxiety and rumination to switching abilities there is an overlap between depression, anxiety, and rumination scores and some of the effects obtained in high ruminators may be partially due to their depression or anxiety levels. Although we controlled for anxiety scores and the effects still remained we did not include measures of social anxiety. Rumination is considered a transdiagnostic process with demonstrated relevance in social anxiety (e.g., Mellings \& Alden, 2000), therefore we cannot fully exclude the possibility that social anxiety played a role.

In sum, the present study clearly shows that the IST measures a relatively stable switching ability and has good test-retest reliability. Moreover, it was found that rumination is characterized by valence-specific switching impairments. 


\section{Acknowledgements}

The research was carried out by Evi De Lissnyder, under the supervision of Rudi De Raedt and Ernst Koster, at the Psychopathology and Affective Neuroscience Lab at Ghent University. The authors would like to thank master students Emmanuel Stein and Lenny Stevens for their help with running the experiment. The authors declare no conflicts of interest. The second author, Evi De Lissnyder, holds a Ph.D fellowship of the Fund for Scientific Research-Flanders (FWO), Belgium. 


\section{References}

Alloy, L. B., Abramson, L. Y., Hogan, M. E., Witehouse, W. G., Rose, D. T., Robinson, M. S, \& Lapkin, J. B. (2000). The Temple-Wisconsin cognitive vulnerability to depression project: lifetime history of Axis I psychopathology in individuals at high and low cognitive risk for depression. Journal of Abnormal Psychology, 109, 403-418.

American Psychological Association (1954). Technical recommendations for psychological tests and diagnostic techniques. Washington, DC:APA.

Banse, R. (2001) Affective priming with liked and disliked persons: Prime visibility determines congruency and incongruency effects. Cognition \& Emotion, 15, 501-520.

Barnett, P. A., \& Gotlib, I. H. (1988). Psychosocial functioning and depression: Distinguishing among antecedents, concomitants, and consequences. Psychological Bulletin, 104, 97-126.

Beck, A.T., Steer, R.A., \& Brown, G.K. (1996). Manual for the Beck Depression Inventory (2nd edn). The Psychological Association : San Antonio, TX.

Beck, A.T., Steer, R.A., \& Garbin, M.G. (1988). Psychometric properties of the Beck Depression Inventory: Twenty-five years of evaluation. Clinical Psychology Review, 8, 77-100.

Bernblum \& Mor, N. (2010). Rumination and emotion-related biases in refreshing information. Emotion, 10, 423-432.

Chambers, R., Lo, B. C. Y., \& Allen, N. B. (2008). The impact of intensive mindfulness training on attentional control, cognitive style, and affect. Cognitive Therapy and Research, 32, 303-322.

Chun, M. M., Golomb, J. D., \& Turk-Browne, N. B. (2011). A taxonomy of external and internal attention. Annual Review of Psychology, 62, 73-101.

Clark, D. A., Beck, A. T., \& Alford, B. A. (1999). Scientific foundations of cognitive theory 
and therapy for depression. John Wiley \& Sons: New York.

Davis, R. N., \& Nolen-Hoeksema, S. (2000). Cognitive inflexibility among ruminators and non-ruminators. Cognitive Therapy and Research, 24, 699-711.

De Lissnyder, E., Derakshan, N., De Raedt, R., \& Koster, E.H.W. (2011). Depressive symptoms and attentional control: An antisaccade study. Cognition \& Emotion, 5, 886897.

De Lissnyder, E., Koster, E.H.W., Derakshan, N., \& De Raedt, R. (2010). The association between depressive symptoms and executive control impairments in response to emotional and non-emotional information. Cognition \& Emotion, 24, 264-280.

De Lissnyder, E., Koster, E.H.W., \& De Raedt, R. (2012). Emotional interference in working memory is related to rumination. Cognitive Therapy and Research, 36, 348-357.

De Raedt, R., \& Koster, E.H.W. (2010). Understanding increasing vulnerability for depression from a cognitive neuroscience perspective: a reappraisal of attentional factors and a new conceptual framework. Cognitive Affective and Behavioral Neuroscience, 10, 50-70.

Donaldson, C., Lam, D. \& Mathews, A. (2007). Attention and Rumination in Major Depression. Behaviour Research and Therapy, 45, 2664-2678.

Garavan, H. (1998). Serial attention within working memory. Memory \& Cognition, 26, 263-276.

Gehring, W. J., Bryck, R. L., Jonides, J., Albin, R. L., \& Badre, D. (2003). The mind's eye, looking inward? In search of executive control in internal attention shifting. Psychophysiology, 40, 572-585.

Goeleven, E., De Raedt, R., Leyman, L., \& Verschuere, B. (2008). The Karolinska Directed Emotional Faces: A validation study. Cognition \& Emotion, 22, 1094-1118.

Gotlib, I. H., \& Hammen, C. I. (2002). Handbook of Depression. Guilford Press: New York. 
Greve, K. W., Stickle, T. R., Love, J. M., Bianchini, K. J., \& Stanford, M. S. (2005). Latent structure of the Wisconsin Card Sorting Test: A confirmatory factor analytic study. Archives of Clinical Neuropsychology, 20, 355-364.

Hertel, P. T. (1997). On the contributions of deficient cognitive control to memory impairments in depression. Cognition \& Emotion, 11, 569-584.

Joormann, J. (2006). The relation of rumination and inhibition: Evidence from a negative priming task. Cognitive Therapy and Research, 30, 149-160.

Joormann, J, \& D'Avanzato C. (2010). Emotion regulation in depression: Examining the role of cognitive processes Cognition \& Emotion, 24, 913-939.

Joormann, J., Dkane, M., \& Gotlib, I. H. (2006). Adaptive and maladaptive components of rumination? Diagnostic specificity and relation to depressive biases. Behavior Therapy, 37, 269-280.

Joormann, J., Levens, S.M., \& Gotlib, I.H. (2011). Sticky thoughts: depression and rumination are associated with difficulties manipulating emotional material in working memory. Psychological Science, 22, 979-983.

Kindt, M., Bierman, D., \& Brosschot, J.F. (1996). Stroop versus Stroop: Comparison of a card format and a single-trial format of the standard color-word Stroop task and the emotional Stroop task. Personality and Individual Differences, 21, 653-661.

Koster, E.H.W., De Lissnyder, E., Derakshan, N., \& De Raedt, R. (2011). Understanding depressive Rumination from a cognitive science perspective: The impaired disengagement hypothesis. Clinical Psychology Review, 31, 138-145.

Linville, P. W. (1996). Attention inhibition: Does it underlie ruminative thought? In R. S. Wyer, Jr. (Ed.), Advances in social cognition (Vol. 9, pp. 121-133). Hillsdale, NJ: Erlbaum. 
Lundqvist, D., Flykt, A., \& Öhman, A. (1998). The Karolinska Directed Emotional Faces (KDEF). Department of Neurosciences, Karolinska Hospital, Stockholm.

Mellings, T.M.B., \& Alden, L.E. (2000). Cognitive processes in social anxiety: the effects of self-focus, rumination and anticipatory processing. Behavior Research and Therapy, 38, 243-257.

Miyake, A., Friedman, N. P., Emerson, M. J., Witzki, A. H., \& Howerter, A. (2000). The unity and diversity of executive functions and their contributions to complex "frontal lobe" tasks: A latent variable analysis. Cognitive Psychology, 41, 49-100.

Monsell, S. (1996). Control of mental processes. In V. Bruce (Ed.), Unsolved mysteries of the mind: Tutorial essays in cognition (pp. 93-148). Hove, UK: Erlbaum.

Nolen-Hoeksema, S. (1991). Responses to depression and their effects on the duration of depressive episodes. Journal of Abnormal Psychology, 100, 569-582.

Nolen-Hoeksema, S., \& Morrow, J. (1991). A prospective study of depression and posttraumatic stress symptoms after a natural disaster: The 1989 Loma Prieta earthquake. Journal of Personality and Social Psychology, 61, 115-121.

Nolen-Hoeksema, S., Wisco, B.E., \& Luybomirsky, S. (2008). Rethinking rumination. Perspectives on Psychological Science, 3, 400-424.

Raes, F., Schoofs, H., Hoes, D., Hermans, D., Van Den Eede, F., \& Franck, E. (2009). 'Reflection' en 'brooding' als subtypes van rumineren: een herziening van de Ruminative Response Scale. [Reflection and brooding as subtypes of rumination: A revision of the Ruminative Response Scale]. Gedragstherapie, 42, 205-214.

Rokke, P., Arnell, K., Koch, M. \& Andrews, J. (2002). Dual-task attention deficits in dysphoric mood. Journal of Abnormal Psychology, 111, 370-379.

Schmukle, S.C. (2005). Unreliability of the dot probe task. European Journal of Personality, $19,595-605$. 
Siegle G.J., Ghinassi F., \& Thase M.E. (2007). Neurobehavioral therapies in the 21st century: Summary of an emerging field and an extended example of cognitive control training for depression. Cognitive Therapy and Research, 31, 235-262.

Spielberger, C. D. (1983). Manual for the State-Trait Anxiety Inventory. Palo Alto, CA: Consulting Psychologists Press.

Treynor, W., Gonzalez, R., \& Nolen-Hoeksema, S. (2003). Rumination reconsidered: A psychometric analysis. Cognitive Therapy and Research, 27, 247-259.

Van der Does, A.J.W. (2002). De Nederlandse Versie van de Beck Depression InventoryTweede Editie. [The Dutch version of the Beck Depression Inventory-Second Edition]. Lisse: Swets \& Zeitlinger.

Van der Ploeg, H. M., Defares, P. B., \& Spielberger, C. D. (2000). Handleiding bij de ZelfBeoordelings Vragenlijst. Een Nederlandstalige bewerking van de Spielberger StateTrait Anxiety Inventory. [Manual for the Dutch State-Trait Anxiety Inventory]. Lisse: Swets and Zeitlinger b.v.

Watkins, E.R. (2008). Constructive and unconstructive repetitive thought. Psychological Bulletin, 134, 163-206

Williams, J. M. G., Watts, F. N., MacLeod, C., \& Mathews, A. (1997). Cognitive psychology and emotional disorders. (2nd ed.). Chichester, England: Wiley.

Whitmer, A. J., \& Banich, M. T. (2007). Inhibition versus switching deficits in different forms of rumination. Psychological Science, 18, 546-553.

Yee Lo, B.C., Lau, S., Cheung, S., \& Allen, N.B. (2012). The impact of rumination on internal attention switching. Cognition \& Emotion, 26, 209-223. 


\section{Footnote}

${ }^{1}$ We use the term switching here in the context of switching between objects and working memory and not task switching. The term switching is also often used in the context of executive functions and cognitive control and is sometimes distinguished from other mental operations such as inhibition and updating. Note that we do not claim that the IST is a "pure measure" of switching as performance is dependent on a number of specific cognitive operations (Greve, Stickle, Love, Bianchini, \& Stanford, 2005). Therefore we describe the IST in relation to its most crucial functional operation, the internal shifting of attention, which maps onto the taxonomy of internal vs. external attention provided by Chun et al. (2011).

${ }^{2}$ Because of the selection procedures, Wilk Shapiro test indicated that brooding and reflection scores were not normally distributed. These variables were transformed into dichotomous variables by creating high and low groups using median-split procedure. Some individuals whose score resembled the median could not be categorized as high or low and were not taken into account in the regression analyses. 
Table 1.

Descriptive statistics and reliability estimates of the reaction time indices of the IST

\begin{tabular}{lcccc}
\hline Reaction time measures (ms) & \multicolumn{2}{c}{ Descriptives } & \multicolumn{2}{c}{ Reliability } \\
& $M$ & $S D$ & Split-half* & Test-retest \\
\hline General switch & 1452 & 240 & & $.876^{* * *}$ \\
General no-switch & 1065 & 133 & & $.713^{* * *}$ \\
General switch cost & 386 & 220 & & $.911^{* * *}$ \\
& & & & \\
Emotion switch & 1499 & 279 & .90 & $.798^{* * *}$ \\
Emotion no-switch & 1083 & 158 & .79 & $.585^{* * *}$ \\
Emotion switch cost & 416 & 275 & .76 & $.858^{* * *}$ \\
Gender switch & 1402 & 229 & .89 & $.832^{* * *}$ \\
Gender no-switch & 1049 & 140 & .81 & $.677^{* * *}$ \\
Gender switch cost & 352 & 206 & .61 & $.853^{* * *}$ \\
& & & & \\
Angry-ne utral & 1551 & 274 & .66 & $.755^{* * *}$ \\
Neutral-angry & 1462 & 300 & .86 & $.674^{* * *}$ \\
Angry-angry & 1089 & 179 & .46 & $.561^{* * *}$ \\
Neutral-neutral & 1072 & 164 & .15 & $.581^{* * *}$ \\
Male-female & 1380 & 258 & .83 & $.769^{* * *}$ \\
Female-male & 1436 & 219 & .54 & $.752^{* * *}$ \\
Male-male & 1055 & 150 & .76 & $.648^{* * *}$ \\
Female-fermale & 1048 & 147 & .42 & $.581^{* *}$ \\
\hline
\end{tabular}

** Correlation is siznificant at the 0.01 level (2-tailed).

* S peaman-Brown correction coefficient 
Table 2

Means and standard deviation for the different trialtypes in the IST of experiment 2.

$\begin{array}{lll}\text { Reaction time measures (ms) } & \text { M } & \text { SD }\end{array}$

Emotion condition

Angry-Angry

1289

252

Neutral-Neutral

1303

271

Angry-Neutral

1697

332

Neutral-Angry

1637

302

Gender condition

Switch gender

1487

300

No-switch gender

1158

240 
Table 3

Zero-order correlations of variables entered in the stepwise regression analyses predicting switch cost in the emotion condition.

\begin{tabular}{|c|c|c|c|c|c|}
\hline & $\begin{array}{l}\text { Switch cost in the } \\
\text { emotion condition }\end{array}$ & $\begin{array}{l}\text { BDI } \\
\text { total }\end{array}$ & $\begin{array}{c}\text { RRS } \\
\text { brooding }\end{array}$ & $\begin{array}{c}\text { RRS } \\
\text { pondering }\end{array}$ & STAI \\
\hline \multicolumn{6}{|c|}{$\begin{array}{l}\text { Switch cost in the } \\
\text { emotion condition }\end{array}$} \\
\hline BDI total & .31 & & & & \\
\hline RRS brooding & $.37^{*}$ & $.61 * *$ & & & \\
\hline RRS pondering & .22 & .25 & $.46 * *$ & & \\
\hline STAI & .25 & $.76^{* *}$ & $.63^{* *}$ & .25 & \\
\hline
\end{tabular}

Note: $* p<.05, * * p<.01$ 
Table 4

Zero-order correlations of variables entered in the stepwise regression analyses predicting valence-specific switch cost.

\begin{tabular}{lccccc}
\hline & $\begin{array}{c}\text { Valence-specific } \\
\text { switching impairment }\end{array}$ & $\begin{array}{c}\text { BDI } \\
\text { total }\end{array}$ & $\begin{array}{c}\text { RRS } \\
\text { brooding }\end{array}$ & $\begin{array}{c}\text { RRS } \\
\text { pondering }\end{array}$ & STAI \\
\hline Valence-specific & & & & & \\
switching impairment & .27 & & & & \\
BDI total & $.38^{*}$ & $.61^{* *}$ & & \\
RRS brooding & .29 & .25 & $.46^{* *}$ & \\
RRS pondering & .25 & $.76^{* *}$ & $.63^{* *}$ & .25 \\
STAI & & & &
\end{tabular}

Note: ${ }^{*} p<.05,{ }^{* *} p<.01$ 


\section{Figure caption list}

Figure 1. An example of a block of items and stimulus display.

Figure 2. Switch cost in the emotion versus gender condition for low and high ruminators in Experiment 2.

Figure 3. Valence-specific effects in the emotional condition for low and high ruminators. The reaction times on the y-axis are based on the reaction times (in ms) on the target trials (Face $n$ ) (e.g., for the angry-neutral bars, the reaction times on the y-axis are the reaction times on the neutral trials that follow angry trials). 
Emotion condition

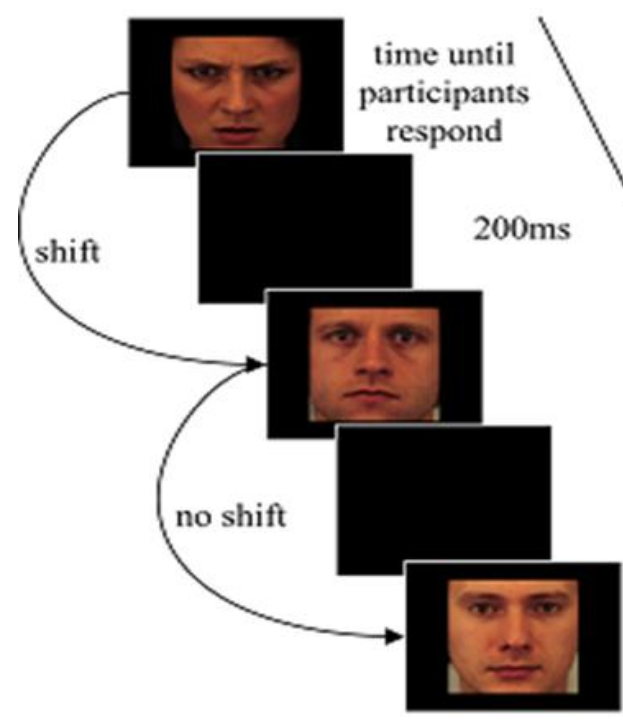

$\ldots$

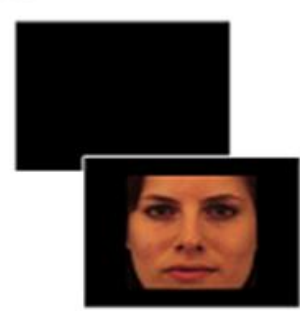

Gender condition

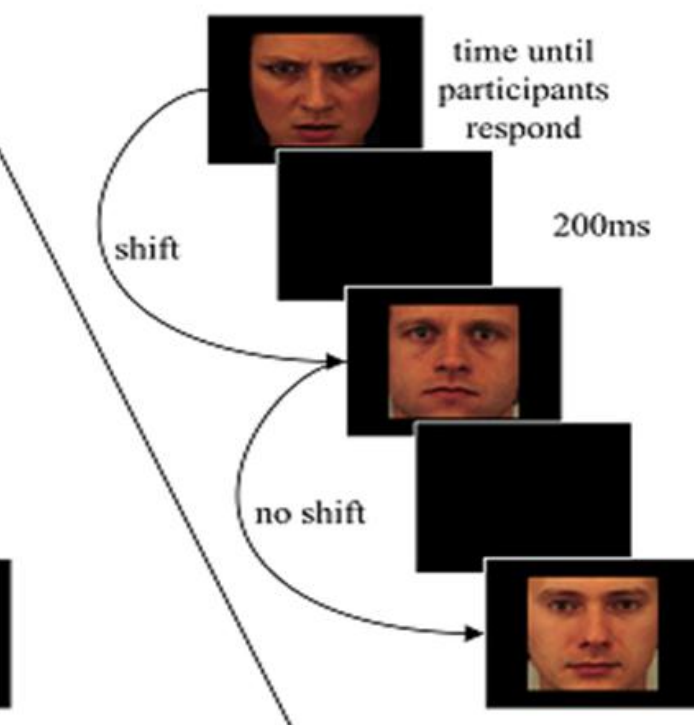

$\ldots$

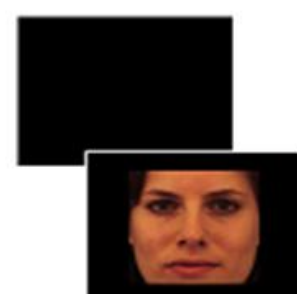


$\square$ Low ruminators

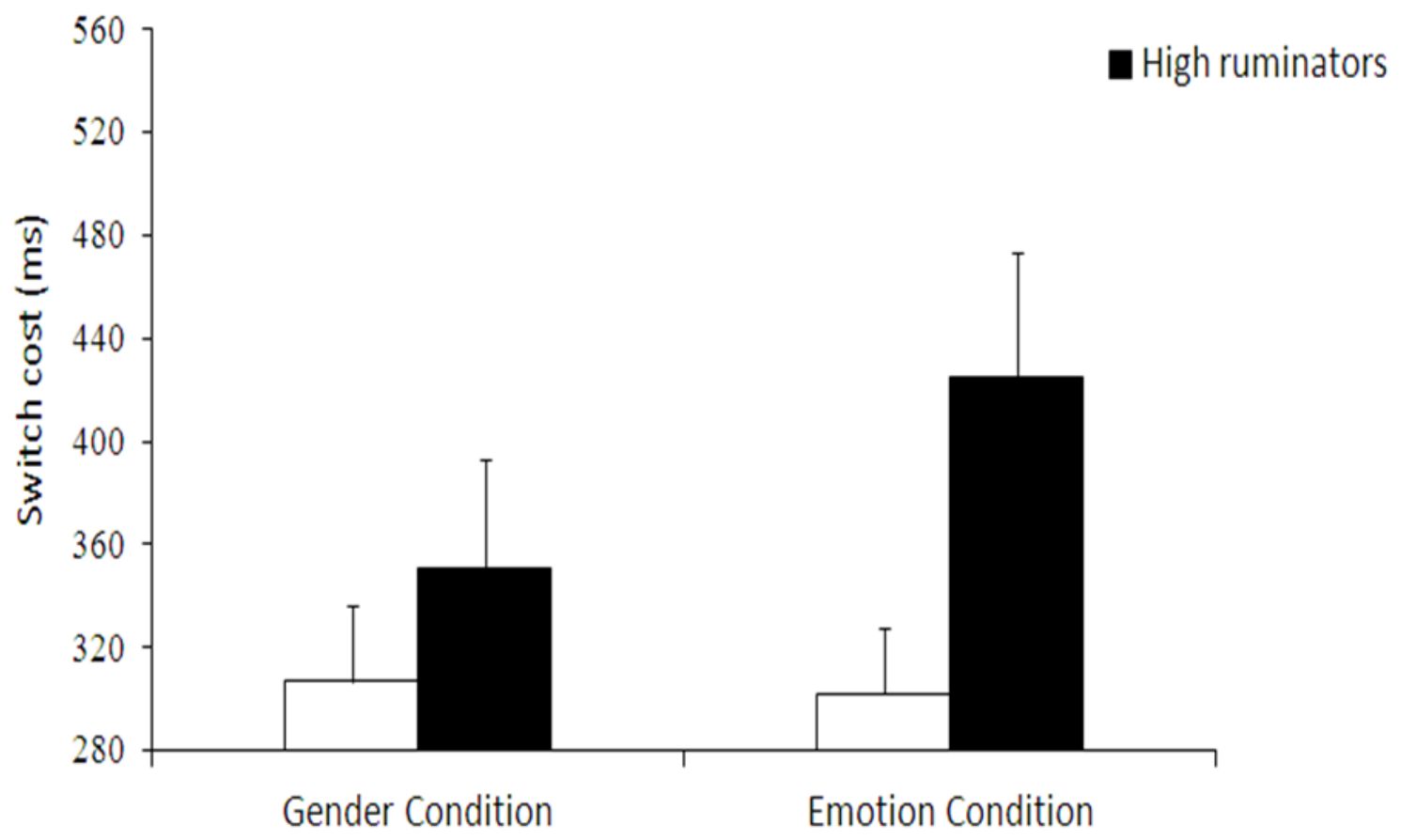




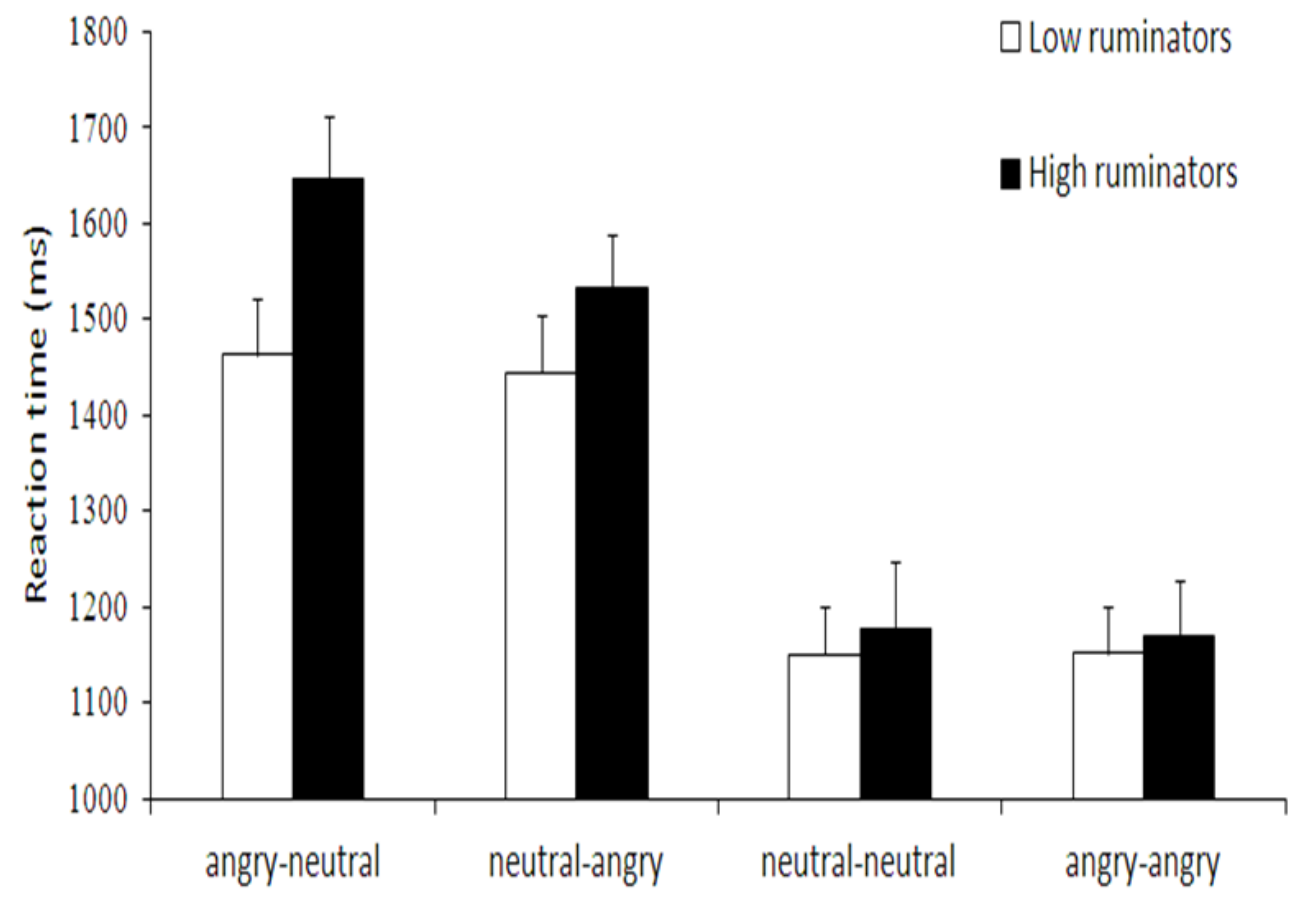

